\title{
Relación entre factores de personalidad y metacognición en una muestra de estudiantes del último semestre de formación de programas de licenciatura en Educación en Colombia
}

\author{
Relationship between personality factors and metacognition in a sample of \\ students during the final semester of Bachelor of Education degree programs in \\ Colombia
}

\author{
Antonio Gutierrez-de Blume (D) \\ Georgia Southern University, Georgia, Estados Unidos \\ Diana Montoya-Londoño (D) \\ Universidad de Caldas, Manizales, Colombia
}

\section{Resumen}

Introducción: las características de la personalidad y la autorregulación de las habilidades de aprendizaje, como la metacognición, tienen una influencia significativa en la vida cotidiana, en el contexto de actividades básicas desde elegir qué hacer, con quién interactuar e incluso hasta en aspectos como qué aprender y a qué ritmo. Objetivo: establecer el valor predictivo de diferentes factores de personalidad, en el conocimiento y la regulación metacognitiva de una muestra de estudiantes de último semestre de formación de diferentes programas de licenciatura en Educación. Método: se trabajó con una muestra de 135 estudiantes colombianos de diferentes programas de pregrado en Educación, quienes diligenciaron el Inventario de Conciencia Metacognitiva para docentes (MAIT) y el instrumento Adjetivos para Evaluar la Personalidad (AEP). Las preguntas de investigación se respondieron utilizando una combinación de estadísticas descriptiva (medias, desviaciones estándar) e inferencial (regresión). Resultados: los hallazgos encontrados indican que la responsabilidad fue la única característica de la personalidad que predijo positivamente el conocimiento declarativo y condicional, así como la planificación y el monitoreo, pero no el conocimiento del procedimiento y la evaluación. Discusión y Conclusiones: los resultados respaldan la necesidad de seguir avanzando en investigaciones que analicen la forma cómo los factores de personalidad afectan las habilidades propias del aprendizaje autorregulado, como la metacognición, a fin de poder impactar la propia capacidad de autorregulación del estudiante, así como su desempeño futuro como docente, en el contexto de una mejor la práctica educativa.

Palabras clave: aprendizaje, metacognición, personalidad, estudiantes universitarios.

\section{Abstract}

Introduction: personality traits and self-regulation of learning skills, such as metacognition, have a significant influence on everyday life in the context of basic activities, from choosing what to do, who to interact with and even on aspects such as what and at what pace to learn. Objective: to establish the predictive value of different personality factors in the knowledge and metacognitive regulation of a sample of students in their final semester of training in different degree programs in Education. Method: we worked with a sample of 135 Colombian students from different undergraduate programs in Education, who completed the Metacognitive Consciousness Inventory for Teachers (MAIT) and the Adjectives for Personality Assessment (AEP) tool. Results: the findings indicate that responsibility was the only personality trait that positively predicted declarative and conditional knowledge, as well as planning and monitoring, but not the knowledge of procedure and evaluation. Discussion and Conclusions: the results support the need to conduct further research about how personality factors affect self-regulated learning skills, such as metacognition, in order to impact on students' own selfregulatory capacity, as well as their future performance as teachers, in the context of improved educational practice.

Keywords: learning, metacognition, personality, university students.
Open Access:

ISSN: $0124-2121$

E-ISSN: $2665-2420$
ARTÍCULO RESULTADO DE INVESTIGACIÓN Copyright $(\subset$

By Educación y Humanismo

Editor:

Dhayana Fernández Matos Universidad Simón Bolívar

\section{Correspondencia:}

Antonio Gutiérrez

agutierrez@georgiasouthern.edu

Recibido: $30-05-2020$

Aceptado: 25-07-2020

En línea desde: 01-10-2020 


\section{Introducción}

En el contexto de las ciencias de la educación se consideran como una línea emergente de investigación los estudios en metacognición (Sawyer, 2014; Zohar \& Dori, 2012), especialmente, el monitoreo metacognitivo del estudiante ha sido un problema central en los estudios de calibración desde el análisis de los niveles de confianza, precisión y disminución del error en la formulación de los juicios metacognitivos (Gutierrez \& Schraw, 2015; Gutierrez de Blume, 2017). Como resultado de las investigaciones realizadas en este campo, se ha indicado que un monitoreo metacognitivo más preciso debería conducir a decisiones de estudio más efectivas y en general, a mejores resultados de aprendizaje (Thiede, Oswalt, Bredefur, Osguthorpe, \& Osguthorpe, 2019). Sin embargo, no ocurre lo mismo en el caso de la metacognición del docente (Kallio et al., 2017), en cuanto el problema del monitoreo metacognitivo se ha investigado con especial énfasis en el caso de los estudiantes, pero realmente muy poco en el caso de los docentes, incluyendo a los maestros en formación, como lo serían los estudiantes de últimos semestres de programas de licenciatura en Educación que se encuentran próximos a graduarse (Wilson \& Bai, 2010).

En consecuencia, se reconoce un énfasis menor en relación con el abordaje investigativo de aspectos como el conocimiento y la regulación metacognitiva en la profesión del docente, a pesar de que se presume que, para formar estudiantes que puedan ser competentes de acuerdo con las demandas de formación del siglo XXI, especialmente a nivel del dominio de habilidades de pensamiento metacognitivo y crítico, se requieren docentes que reflexionen acerca de lo que enseñan y de cómo lo enseñan, y que autorregulen su enseñanza en consecuencia (Joseph, 2009; Prytula, 2012; Stephenson, 2008), ideal de formación que debería estar presente desde las Facultades de Educación, a fin de privilegiar en la formación de las nuevas generaciones de maestros, el desarrollo del conocimiento y la regulación metacognitiva frente a su enseñanza desde el mismo inicio de la carrera docente, en cuanto se ha considerado que, promover en el maestro la capacidad para autorregular el aprendizaje es fundamental para el crecimiento profesional de los docentes durante toda su carrera, y de manera especial, desde el inicio de la formación, dado que, el desarrollo de la propia metacognicion frente a la enseñanza, parece fundamental en la posibilidad futura que tenga el maestro de favorecer el desarrollo de procesos metacognitivos en los estudiantes a cargo (Kramarski \& Michalsky, 2009; Prytula, 2012).

De igual forma, son relativamente pocos los estudios en los que los investigadores se han interesado en estimar el valor de predictores no cognitivos en el éxito escolar, entre los que se encuentran la importancia de diferentes factores de personalidad, en el desempeño metacognitivo, más allá de la realización de algunos análisis sobre la implicación de medidas de ajuste social y psicológico, así como en el estudio de algunos 
aspectos de las diferencias individuales en la formulación de juicios metacognitivos en estudiantes de secundaria y en reclutas de la fuerza aérea, trabajos en los que se ha encontrado que los rasgos de personalidad y la capacidad cognitiva se relacionan con la precisión de la autoevaluación y que la confianza en la respuesta que tiene la persona en una prueba cognitiva correlaciona con otras medidas de autoconfianza, como el autoconcepto y la autoeficacia (Pallier, et al., 2002; Stankov, Morony, \& Ping Lee, 2014).

En general puede indicarse que aunque existe una gran tradición investigativa, en torno al monitoreo metacognitivo del estudiante, desde la cual se han derivado hallazgos respecto al efecto de diferentes procesos de intervención (Gutierrez \& Schraw, 2015; Nietfeld \& Schraw, 2002), se ha tenido un desarrollo mucho menor del campo investigativo de la metacognición del docente (Prytula, 2012; Wilson \& Bai, 2010).

El abordaje del problema de la metacognición se ha realizado desde una perspectiva que privilegia el estudio de diferentes habilidades propias del conocimiento y la regulación metacognitiva, sin considerar necesariamente la influencia de variables más cálidas en el actuar metacognitivo, como lo serían factores como la motivación, las expectativas o la personalidad en las posibilidades de desempeño metacognitivo de la persona. Al respecto, algunos investigadores consideran que, este hecho probablemente puede estar asociado con el marcado énfasis de los estudios de metacognición en una perspectiva de cognición fría, en contraste con la necesidad de analizar la influencia en el desempeño metacognitivo de variables propias de una postura de la cognición más cálida, entre las que pueden encontrarse el estilo parental, la motivación, las emociones y la personalidad (Burson, Larrick \& Klayman, 2006; Stankov, Morony \& Ping Lee, 2014).

El abordaje de la relación entre factores de personalidad y metacognición, puede aportar elementos importantes para la explicación del proceso de autorregulación del aprendizaje de la persona en el rol del docente, lo cual representa el interés de la presente investigación. Los factores de personalidad pueden entenderse como disposiciones relativamente estables de una persona a ciertos patrones de cognición, emoción y comportamiento (Hogan, Hogan \& Roberts, 1996; Ozturk, 2020). Desde este enfoque algunos autores han reconocido una estructura de cinco factores entre los que se encuentran las dimensiones de amabilidad, estabilidad emocional (o neuroticismo), conciencia o responsabilidad, extraversión y apertura a la experiencia (o intelecto) (Goldberg et al., 2006; Kim, Jörg \& Klassen, 2019; McCrae \& Costa, 1987).

Algunos investigadores han señalado que la operacionalización de estos factores de personalidad puede incluir la tendencia hacia algunas habilidades de autorregulación del aprendizaje (Bidjerano \& YunDai, 2007; Blair, Calkins \& Kopp, 2010). Entre estas posturas están las que reconocen que la conciencia o responsabilidad se asocia con la capacidad para planificar, organizar y persistir en las actividades de aprendizaje y que, de igual forma, las personas con altas puntuaciones en el factor de apertura se evidencian como dispuestas hacia experiencias de aprendizaje desafiantes (Bidjerano \& YunDai, 2007; 
McCrae \& Costa, 1987). Al mismo tiempo, otros investigadores han señalado que los estudiantes con mayores habilidades de autorregulación presentan menor neuroticismo y valores más altos en los factores de extraversión, conciencia, amabilidad y apertura a las experiencias (Dörrenbächer \& Perels, 2016).

Entre los antecedentes que se reportan en el abordaje del conocimiento y la regulación metacognitiva en el contexto de la enseñanza, se destacan algunos estudios descriptivos en los que se han caracterizado muestras de docentes con diferentes niveles de formación (últimos semestres de pregrado, profesional, y posgradual), en diferentes áreas de dominio (inglés, biología, matemáticas, etc.) y en diferentes contextos culturales (Turquía, Irán, Indonesia, Finlandia, entre otros países) a partir del uso de instrumentos de evaluación de autorreporte, entre los que se destacan el Inventario de Conciencia Metacognitiva General (MAI) (Schraw \& Dennison, 1994), el Inventario de Conciencia Metacognitiva para Docentes (MAIT) (Balcikanli, 2011), una versión abreviada del MAIT (MAIT-18) (Kallio et al., 2017) y el Inventario de Metacognición del Maestro (TMI) (Jiang, Ma \& Gao, 2016). Entre los resultados que han reportado estos estudios, se ha señalado que el nivel de conciencia metacognitiva de los maestros influye en su pensamiento reflexivo y sobre sus experiencias de enseñanza práctica (Adadan \& Oner, 2018). De igual modo, se reporta que tanto las medidas metacognitivas como las de autoeficacia afectan el rendimiento académico de los docentes en ejercicio (Ghonsoolya, Hassan Khajavyb, \& MohagheghMahjoobi, 2014).

En otros estudios se ha indicado una baja correlación entre la capacidad metacognitiva y los resultados de las pruebas estandarizadas de competencia docente aplicada a maestros (Ardi, Fadilah \& Ichsani, 2018).

En general, diferentes estudios concluyen que el conocimiento, la regulación y capacidad de autoconciencia respecto al proceso de enseñanza del docente, así como el conocimiento de las propias habilidades metacognitivas, es un aspecto que potencialmente puede beneficiar el aprendizaje de los estudiantes (Jiang et al., 2016), dado que los maestros necesitan conocer sus propias habilidades en esta área primero, para luego poder ayudar a sus estudiantes a mejorar sus habilidades para autorregular su aprendizaje (Kallio et al., 2017).

Teniendo en cuenta que hay poca tradición investigativa sobre la exploración de la relación entre las características de la personalidad y las variables metacognitivas, se considera que el presente estudio llenará este vacío y aumentará la explicación de esta relación dinámica. Esta investigación tuvo como objetivo establecer el valor predictivo de diferentes factores de personalidad, en el conocimiento y la regulación metacognitiva de una muestra de estudiantes de último semestre de formación de diferentes programas de licenciatura en Educación. 


\section{Pertinencia del estudio}

El análisis de la relación entre factores de personalidad y componentes de la metacognición, como lo son el conocimiento y la regulación metacognitiva, como el que se comunica en el presente artículo, se considera pertinente en cuanto representa un aporte en el desarrollo de nuevas líneas de trabajo en el campo de los estudios en metacognición, que incluyen el análisis de nuevas variables, entre ellas, la personalidad. Este aporte resulta relevante, en cuanto los procesos de intervención que se realizan tanto a nivel de la metacognición, como del aprendizaje autorregulado, evidencian resultado en ocasiones poco concluyentes, en los que los estudiantes parecen manifestar cierta resistencia al cambio, que podría ser mejor explicada por variables como la personalidad. (Bol, Hacker, O'Shea \& Allen, 2005; Zimmerman \& Moylan, 2009). El presente estudio representa un aporte en esta búsqueda de explicación.

A su vez, hacer este trabajo en una institución colombiana, además de aquellos que reclutan muestras de países de habla inglesa, como ha sido el caso en muchos estudios en el campo de la metacognición, contribuye a mejorar la generalización de los resultados de la investigación a muestras de habla hispana.

\section{Pregunta de investigación e hipótesis}

¿Cuál es el valor predictivo de diferentes factores de personalidad en el conocimiento y la regulación metacognitiva de una muestra de estudiantes de último semestre de formación de programas de licenciatura en Educación?

\section{Hipótesis}

En el momento actual, la investigación sobre la relación entre constructos cognitivos y rasgos de personalidad no ha sido concluyente, ni clara (p.ej., Bidjerano \& YunDai, 2007; Blair, Calkins \& Kopp, 2010; Dörrenbächer \& Perels, 2016). Es en el contexto de esta falta de claridad, que en la presente investigación se propuso una hipótesis no direccional más general a la pregunta de investigación, en lugar de proponer una direccional más específica. Así se esperaba que los factores de personalidad de interés pudieran predecir significativamente las habilidades metacognitivas evaluadas. Se esperaba que el neuroticismo, por ejemplo, predijera negativamente las habilidades metacognitivas, mientras que se esperaba que factores como la extraversión, la amabilidad, la responsabilidad y la apertura predijera positivamente las habilidades metacognitivas. 


\section{Método}

\section{Participantes y muestra}

Los participantes fueron 156 estudiantes de pregrado de diferentes programas de licenciatura en Educación de una universidad pública en el centro de Colombia, que se encontraban cursando su último semestre de carrera durante el año 2019. Así, la muestra estuvo compuesta por 88 hombres y 66 mujeres. Las especialidades de formación de los estudiantes implicaron los programas de artes escénicas $(n=12)$, biología y química ( $n=$ $18)$, ciencias naturales $(n=2)$, historia y geografía $(n=23)$, educación física $(n=40)$, filosofía $(n=11)$, idiomas modernos $(n=14)$, lengua castellana $(n=1)$, música $(n=15)$ y no declarado $(n=19)$. La selección de la muestra se realizó de manera intencional, de acuerdo con los lineamientos metodológicos de un muestreo por conveniencia, dado que los participantes estaban inscritos cursando su carrera profesional en la universidad en la que labora uno de los autores y, una vez, que estos fueron informados acerca de la investigación, ellos se interesaron y aceptaron participar voluntariamente en el estudio. Al estar en su último semestre de carrera, se les consideró docentes en formación.

Todos los estudiantes estuvieron matriculados en la universidad durante el año 2019 y cumplieron con los siguientes criterios de inclusión: edad entre 20 a 30 años; ausencia de alteraciones psicológicas y/o psiquiátricas o historial de repitencia, o rezago escolar de acuerdo con el reporte del historial clínico y académico registrado en la sección de prácticas profesionales de la universidad; y se contó con la firma del consentimiento informado por parte del estudiante.

\section{Materiales e instrumentos}

MAIT. Inventario de Conciencia Metacognitiva para Docentes (Balcikanli, 2011). Este instrumento es una versión adaptada al contexto de la enseñanza, con base en el inventario clásico para la evaluación de la conciencia metacognitiva en adultos (MAI) (Schraw \& Dennison, 1994). El alfa de Cronbach reportado para la prueba varía desde 0.79 para el caso de la habilidad de evaluación hasta 0.85 para el caso del conocimiento declarativo (Balcikanli, 2011; Mai, 2015; Palantis et al., 2017).

AEP. Listado de adjetivos para evaluar la personalidad (Ledesma, Sánchez \& DíazLázaro, 2011; Sánchez \& Ledesma, 2013). Es un instrumento que se estructura a partir del modelo de los cinco grandes factores (Goldberg, 1992; Goldberg, et al., 2006) como un formato compuesto por un listado de 67 adjetivos descriptores de los rasgos de personalidad. El alfa de Cronbach para la prueba oscila entre 0.75 para el factor de apertura hasta 0.84 para el caso del factor de amabilidad y 0.85 para el factor de neuroticismo (Ledesma, Sánchez \& Díaz-Lázaro, 2011; Sánchez \& Ledesma, 2013). 


\section{Procedimiento}

Para la recolección de la información, se convocó en dos momentos a los estudiantes de diferentes programas de licenciatura en educación, que se encontraban cursando su último semestre de carrera durante el año 2019. En este espacio, los estudiantes conocieron los fundamentos de la investigación, a partir de la descripción hecha por el equipo de trabajo, luego de lo cual leyeron y firmaron el correspondiente consentimiento informado bajo la asesoría de una de las docentes investigadoras. Posteriormente, los estudiantes diligenciaron los instrumentos 'Inventario de Conciencia Metacognitiva para Docentes' (MAIT) (Balcikanli, 2011) y el 'Listado de adjetivos para evaluar la personalidad' (AEP) (Ledesma, Sánchez, \& Díaz-Lázaro, 2011; Sánchez \& Ledesma, 2013). Ambas pruebas fueron aplicadas de manera grupal. Asimismo, es importante señalar que, en la aplicación de los instrumentos, se tuvieron en cuenta los lineamientos éticos planteados desde la Resolución 008430del 4 de Octubre/1993 en su artículo 11 para los estudios considerados como de riesgo mínimo con seres humanos en el país (Ministerio de Salud, República de Colombia, 1993) y para la protección del anonimato y de los datos de investigación consignados en la ley 1581 (Congreso de la República de Colombia, 2012).

\section{Análisis de los datos}

Primero, se examinaron los datos recolectados para detectar valores atípicos univariantes y se hicieron pruebas (p.ej., normalidad, homogeneidad de varianza, homocedasticidad) para establecer los supuestos estadísticos (p.ej., la linealidad, la homocedasticidad, la normalidad univariada y la falta de colinealidad) necesarios antes de proceder con el análisis de regresión empleando el paquete estadístico para las ciencias sociales (SPSS) versión 23.

De la muestra general, se incluyeron en este proceso de análisis solo los 145 participantes que diligenciaron todos los instrumentos. Luego, los resultados reportados en 10 casos se consideraron atípicos a través de análisis de gráficos de caja y bigotes, que demostraron que estos datos eran más de tres desviaciones estándar más allá de las medias y, en consecuencia, fueron eliminados de cualquier análisis para informar los resultados más exactos y precisos. Finalmente, se trabajó con una muestra de 135 casos completos para los análisis posteriores. Los datos cumplieron con todos los supuestos estadísticos requeridos, incluida la linealidad, la homocedasticidad, la normalidad univariada y la falta de colinealidad y, por lo tanto, el análisis de datos se continuó sin hacer ningún ajuste estadístico adicional.

La pregunta principal considerada en la presente investigación fue respondida realizando una serie de regresiones estándar/simultáneas de mínimos cuadrados ordinarios (MCO). En cada uno de los modelos de regresión, las variables de personalidad de la prueba 'AEP' (extraversión, amabilidad, responsabilidad, neuroticismo y apertura) sirvieron como predictores y cada una de las variables de metacognición (conocimiento 
metacognitivo: declarativo, de procedimiento y condicional; y regulación metacognitiva: planificación, monitoreo y evaluación) sirvieron como criterio en cada uno de los análisis respectivamente. Se empleó el ajuste de Bonferroni a la significancia estadística para corregir la inflación familiar de la tasa de error Tipo I, dadas las múltiples regresiones de MCO, valor $p$ real $=.008(.05 / 6)$. La importancia práctica de los hallazgos encontrados, 0 el tamaño del efecto, se informó como el coeficiente de correlación múltiple al cuadrado, $R^{2}$. De igual forma, Cohen (1988) proporcionó las siguientes pautas interpretativas para el tamaño del efecto, $R^{2}$ : .01-.49 como pequeño; .50-.79como medio, y $\geq .80$ como grande.

\section{Resultados}

Las estadísticas descriptivas y los coeficientes de confiabilidad de consistencia interna, a de Cronbach, para la muestra por variable se presentan en la Tabla 1 y la Tabla 2 muestra correlaciones bivariadas de orden cero para todas las variables de interés en este estudio.

Tabla 1.

Estadística descriptiva y coeficientes de confiabilidad de consistencia interna para las variables de personalidad, conocimiento y regulación metacognitiva

\begin{tabular}{lccc}
\hline Variable & $M$ & $S D$ & $\alpha$ \\
\hline Extraversion $^{\text {a }}$ & 3.72 & 0.72 & 0.82 \\
Amabilidad $^{\mathrm{a}}$ & 4.17 & 0.50 & 0.80 \\
Responsabilidad $^{\mathrm{a}}$ & 4.02 & 0.47 & 0.79 \\
Neuroticismo $^{\mathrm{a}}$ & 2.51 & 0.58 & 0.84 \\
Apertura $^{\mathrm{a}}$ & 3.64 & 0.59 & 0.84 \\
Conocimicnto Dcclarativo $^{\mathrm{b}}$ & 4.19 & 0.53 & 0.74 \\
Conocimiento Procedural $^{\mathrm{b}}$ & 4.09 & 0.52 & 0.76 \\
Conocimiento Condicional $^{\mathrm{b}}$ & 4.16 & 0.48 & 0.78 \\
Planeación $^{\mathrm{b}}$ & 4.18 & 0.55 & 0.77 \\
Monitoreo $^{\mathrm{b}}$ & 4.22 & 0.50 & 0.81 \\
Evaluación $^{\mathrm{b}}$ & 4.24 & 0.46 & 0.75 \\
\hline
\end{tabular}

${ }^{\mathrm{a}}$ Variables de personalidad $\quad{ }^{\mathrm{b}}$ Variables metacognitivas

$N=135$

Fuente: elaboración propia. 
Tabla 2.

Matriz de correlación bivariada de orden cero para las variables de personalidad y metacognitivas

\begin{tabular}{|c|c|c|c|c|c|c|c|c|c|c|c|}
\hline Variable & 1 & 2 & 3 & 4 & 5 & 6 & 7 & 8 & 9 & 10 & 11 \\
\hline 1. DK & - & $.62 * *$ & $.61 * *$ & $.37 * *$ & $.39 * *$ & $.34 * *$ & $.28 * *$ & $.18 *$ & $.32 * *$ & -.12 & $.23 * *$ \\
\hline 2. PK & & - & $.67 * *$ & $.47 * *$ & $.48^{* *}$ & $.35^{* *}$ & $.20^{*}$ & $.25 * *$ & $.24 * *$ & -.05 & $.22 * *$ \\
\hline 3. CK & & & - & .49 *** & $.56^{* *}$ & $.39 * *$ & $.32 * *$ & $.36^{* * *}$ & $.33^{* *}$ & $-.16^{*}$ & $.27^{* * *}$ \\
\hline 4. Planeación & & & & - & $.62 * *$ & $.39 * *$ & $.17^{*}$ & $.25 * *$ & $.44 * *$ & $-.16^{*}$ & .15 \\
\hline 5. Monitorco & & & & & - & $.70 * *$ & $.25^{* *}$ & $.32 * *$ & $.35^{* *}$ & -.12 & .14 \\
\hline 6. Evaluación & & & & & & - & .05 & $.30 * *$ & $.26^{* *}$ & -.04 & .09 \\
\hline 7. Extraversión & & & & & & & - & $.29 * *$ & $.24 * *$ & $-.24^{* *}$ & $34 * *$ \\
\hline 8. Amabilidad & & & & & & & & - & $.46^{* *}$ & $-.23 * *$ & $.40^{* *}$ \\
\hline $\begin{array}{l}9 . \\
\text { Responsibilidad }\end{array}$ & \multicolumn{10}{|c|}{ Rcsponsibilidad } & $.22 * *$ \\
\hline 10. Neuroticismo & & & & & & & & & & - & -.08 \\
\hline 11. Apertura & & & & & & & & & & & - \\
\hline$* * p<.01 * p$ & & & & & & & & & & & \\
\hline$N=135$ & & & & & & & & & & & \\
\hline
\end{tabular}

Fuente: elaboración propia.

Los coeficientes de correlación producto-momento de Pearson variaron de débil, $r=$ .04, a fuerte, $r=.70, \mathrm{y}$ todos estaban en la dirección teóricamente esperada: todas las correlaciones fueron negativas con neuroticismo y positivas para todas las demás correlaciones bivariadas.

Los resultados de los análisis de regresión MCO se resumen en la Tabla 3. 
Tabla 3.

Resultados de regresión de mínimos cuadrados ordinarios para variables de personalidad y metacognitivas

\begin{tabular}{|c|c|c|c|c|}
\hline Predictor & $B \quad\left(\mathrm{CJ}_{95 \%}\right)$ & $\beta$ & $t$ & $p$ \\
\hline \multicolumn{5}{|c|}{ Conocimiento Declarativo } \\
\hline Exlraversión & $0.15(-0.01,0.30)$ & 0.19 & 1.89 & $0.06^{\text {"s }}$ \\
\hline Amabilidad & $0.11(-0.08,0.37)$ & 0.09 & 0.88 & $0.38^{1 \mathrm{~s}}$ \\
\hline Responsibilidad & $0.32(0.09,0.55)$ & 0.30 & 2.74 & $0.01 * *$ \\
\hline Neuroticismo & $0.01(-.156,0.18)$ & 0.01 & 0.12 & 0.91 us \\
\hline Apertura & $0.14(-0.03,0.31)$ & 0.16 & 1.62 & $0.10^{\mathrm{m}}$ \\
\hline \multicolumn{5}{|c|}{ Conocimicnto procedural } \\
\hline Extraversión & $0.07(-0.08,0.23)$ & 0.09 & 0.92 & $0.36^{1 \mathrm{~s}}$ \\
\hline Amabilidad & $0.10(-0.15,0.34)$ & 0.09 & 0.77 & $0.44^{\mathrm{this}}$ \\
\hline Responsibilidad & $0.18(-0.06,0.42)$ & 0.16 & 1.47 & 0.15 us \\
\hline Neuroticismo & $-0.05(-0.12,0.22)$ & -0.05 & -0.54 & $0.60^{\mathrm{ns}}$ \\
\hline$\Lambda$ pertura & $0.11(-0.06,0.29)$ & 0.13 & 1.30 & $0.20^{\mathrm{tis}}$ \\
\hline \multicolumn{5}{|c|}{ Conocimiento Condicional } \\
\hline Fxtraversión & $0.13(-0.01,0.27)$ & 0.18 & 1.88 & $0.06^{\text {tis }}$ \\
\hline Amabilidad & $0.16(-0.06,0.37)$ & 0.15 & 1.42 & $0.16^{\mathrm{ns}}$ \\
\hline Responsibilidad & $0.28(0.03,0.39)$ & 0.29 & 2.66 & $0.01 * *$ \\
\hline Neuroticismo & $-0.01(-0.16,0.14)$ & -0.01 & -0.10 & $0.92^{\text {tis }}$ \\
\hline Apcrtura & $0.10(-0.06,0.25)$ & 0.12 & 1.25 & $0.21^{\mathrm{ns}}$ \\
\hline \multicolumn{5}{|c|}{ Planeación } \\
\hline Extraversión & $0.02(-0.14,0.17)$ & 0.02 & 0.20 & $0.85^{\text {ns }}$ \\
\hline Amabilidad & $0.02(-0.26,0.22)$ & 0.02 & 0.15 & $0.88^{\text {us }}$ \\
\hline Responsibilidad & $0.48(0.25,0.70)$ & 0.42 & 4.01 & $0.001^{\div *}$ \\
\hline Neuroticismo & $-0.03(-0.19,0.13)$ & -0.04 & -0.38 & $0.70^{\mathrm{ss}}$ \\
\hline Apcrtura & $0.06(-0.10,0.23)$ & 0.07 & 0.72 & $0.47^{\mathrm{as}}$ \\
\hline \multicolumn{5}{|c|}{ Monitoreo } \\
\hline Extraversión & $0.11(-0.04,0.25)$ & 0.14 & 1.46 & $0.15^{\mathrm{si}}$ \\
\hline Amabilidad & $0.15(-0.07,0.38)$ & 0.15 & 1.36 & $0.18^{\mathrm{ns}}$ \\
\hline Responsibilidad & $0.24(0.03,0.46)$ & 0.24 & 2.21 & $0.02^{* *}$ \\
\hline Neuroticismo & $-0.02(-0.13,0.18)$ & -0.03 & -0.31 & $0.76^{\mathrm{ms}}$ \\
\hline Apertura & $0.01(-0.16,0.15)$ & $0.0 \mathrm{l}$ & 0.06 & $0.96^{\mathrm{ns}}$ \\
\hline \multicolumn{5}{|c|}{ Evaluación } \\
\hline Extraversión & $0.04(-0.19,0.10)$ & 0.06 & 0.63 & $0.53^{\text {as }}$ \\
\hline Amabilidad & $0.25(-0.02,0.47)$ & 0.20 & 1.85 & $0.05^{\mathrm{ss}}$ \\
\hline Responsibilidad & $0.16(-0.05,0.38)$ & 0.16 & 1.51 & $0.13^{\text {गк }}$ \\
\hline Neuroticismo & $-0.04(-0.11,0.19)$ & -0.05 & -0.52 & $0.61^{\mathrm{as}}$ \\
\hline Apertura & $0.02(-0.18,0.13)$ & 0.03 & 0.25 & $0.80^{\mathrm{ns}}$ \\
\hline
\end{tabular}

$N=135 \quad * p<.05 \quad * * p<.01 \quad{ }^{\text {II* No- Significativo }}$

$B^{\prime}=$ Coeficientes de regresión no estandarizados $95 \%$ Intervelo de confianza (CI I55 )

$\beta=$ Coeficientes de regresión estandarizados

Fuente: elaboración propia.

Los resultados revelaron que solo los modelos con conocimiento declarativo, $F_{(5,130)}=$ 4.84, $p=.001, R^{2}=.17$; conocimiento condicional, $F_{(5,130)}=5.80, p<.001, R^{2}=.21$; 
planificación, $F_{(5,130)}=5.59, p<.001, R^{2}=.20$; y monitoreo, $F_{(5,130)}=4.44, p=.001, R^{2}$ $=.16$, como resultados, alcanzaron significación estadística después del ajuste de Bonferroni al valor $p(.05 / 6=.008)$. Ni el modelo con conocimiento de procedimiento, $\mathrm{ni}$ la evaluación alcanzaron significación estadística, todos los valores de $p \geq 0.03$.

Resulta de interés que el factor de responsabilidad fue la única característica de personalidad que predijo significativa y positivamente el conocimiento declarativo y condicional, como variables de conocimiento metacognitivo, así como la planificación y el monitoreo, como variables de regulación metacognitiva. Este efecto fue particularmente pronunciado para la planificación, en la cual el coeficiente de regresión estandarizado fue 0.42 lo que indica que, por cada unidad de aumento de responsabilidad, la planificación aumenta en 0.42 de una desviación estándar. En estos modelos significativos, cabe mencionar que, aunque no sea estadísticamente significativo, puede señalarse que, el siguiente mejor predictor además de la responsabilidad, fue el factor de extraversión, con la excepción del modelo con planificación como criterio.

\section{Discusión}

El presente estudio tuvo como objetivo establecer el valor predictivo de diferentes factores de personalidad (extraversión, amabilidad, responsabilidad, neuroticismo y apertura) en las habilidades metacognitivas de conocimiento (declarativo, procesal y condicional) y regulación (planificación, monitoreo y evaluación) de una muestra de estudiantes de último semestre de formación de diferentes programas de licenciatura en Educación

En relación con los hallazgos encontrados, puede indicarse que, las correlaciones bivariadas de orden cero revelaron que todas las correlaciones estaban en la dirección teóricamente esperada, en la medida en que las variables metacognitivas estaban positivamente relacionadas con la extraversión, la amabilidad, la responsabilidad y la apertura, pero negativamente con el neuroticismo. Además, la mayoría de las correlaciones fueron estadísticamente significativas y positivas, aunque de pequeñas a moderadas en tamaño.

Con respecto a la pregunta de investigación, los datos respaldaron la hipótesis propuesta. Curiosamente, dentro de los factores de personalidad que fueron evaluados, solo el factor de la responsabilidad fue un predictor positivo y significativo de las habilidades metacognitivas de conocimiento, a saber, el conocimiento declarativo y condicional, así como también, de las habilidades de la regulación, como la planificación y el monitoreo. En general, a medida que aumenta la responsabilidad autoinformada de los participantes, también aumenta el conocimiento declarativo, el conocimiento condicional, la planificación y el monitoreo. Ninguno de los otros factores de la personalidad se acercó a ser predictores significativos de las habilidades metacognitivas. 
El establecimiento de relaciones entre diversos factores de personalidad, con el conocimiento y la regulación metacognitiva del docente, representa en sí mismo, una vía innovadora de investigación (Sawyer, 2014), pues implica reconocer la importancia que tiene en el quehacer profesional del maestro, el desarrollo de la capacidad para conocer y regular los propios recursos cognitivos, y metacognitivos, en función de favorecer la autorregulación del aprendizaje de los estudiantes (Kallio et al., 2017), así como, la reflexión y el ajuste de la enseñanza con base en el proceso de monitoreo metacognitivo del docente (Sanmartí, 2008). De esta manera, algunos autores han considerado que la enseñanza metacognitiva implica que los maestros piensen en cómo el proceso de enseñanza activará y desarrollará los recursos metacognitivos de los estudiantes, o que piensen en su pensamiento desde su propia condición como aprendices (Mai, 2015; Rahman, 2011).

Desde diferentes estudios se considera que el modelo de los cinco grandes factores de personalidad (MCF) evidencia características universales (Costa, Terracciano \& McCrae, 2001; Maltby, Day \& Macaskill, 2007; Sánchez \& Ledesma, 2007), y se ha reconocido su importancia en el establecimiento de relaciones con otras variables de interés en la enseñanza y el aprendizaje, como el enfoque de aprendizaje (superficial o profundo), las habilidades metacognitivas (generales y de dominio específico), el locus de control (externo e interno), las creencias epistemológicas y los estilos de aprendizaje (Batteson, Tormey \& Ritchiec, 2014; Komarraju, Karau, Schmeck \& Avdic, 2011). En la presente investigación se evidenció el valor predictivo del factor de la responsabilidad en las habilidades metacognitivas de conocimiento (declarativo y condicional) y de regulación (planificación y monitoreo) en la muestra de los estudiantes de último semestre de programas de formación de maestros que fueron evaluados.

Así, el factor de responsabilidad o conciencia fue el mejor predictor de las habilidades metacognitivas de los docentes en formación, este hallazgo puede explicarse dado que este factor de personalidad incluye características como competencia, organización, autodisciplina, escrupulosidad y esmero (Goldberg, 1993), todos estos aspectos se consideran esenciales en el desarrollo del conocimiento y la regulación metacognitiva (Batteson et al., 2014), a la vez, que se reconoce que tienen importancia en el ejercicio docente para el desarrollo de una enseñanza eficiente y un aprendizaje exitoso.

Al respecto, algunos investigadores han señalado que la propia metacognición del maestro afecta significativamente el proceso de enseñanza que ejerce y las posibilidades de aprendizaje del estudiante (Fathima, Sasikumar \& Roja, 2014; Jiang et al., 2016), mientras que otros han planteado que es la conciencia metacognitiva de los maestros y la comprensión de las estrategias y habilidades requeridas para la enseñanza y el aprendizaje lo que más parece incidir en su práctica y en los resultados de aprendizaje de sus estudiantes (Zohar, 2006). 
A su vez, la extraversión en la presente investigación fue el segundo factor predictor de las habilidades metacognitivas de los docentes en formación, hallazgo que coincide con otros estudios que han reportado que las personas mucho más cálidas, más sociales, imaginativas, abiertas y creativas tienen más probabilidades de ser metacognitivamente más conscientes de sus habilidades reguladoras y del uso de estrategias al participar en una actividad de enseñanza o aprendizaje (Öz $\left.z^{a}, 2016\right)$.

\section{Implicaciones para la teoría, la investigación y la práctica}

Avanzar la investigación de la relación dinámica entre los factores de aprendizaje "fríos", como la cognición y la metacognición, y los que se consideran "cálidos", como la personalidad, es de especial importancia para los investigadores y profesionales. En la primera mitad del período histórico que avanza, en el estudio de las ciencias del aprendizaje, los investigadores dedicaron la mayor parte de su tiempo a examinar los factores cognitivos y metacognitivos que influyen en los resultados del aprendizaje, mientras que, las construcciones más cálidas, como la motivación y la personalidad, han recibido tradicionalmente menos atención. Sin embargo, no hace falta decir que las características de la personalidad tienen una profunda influencia no solo en la forma como el maestro se conoce y regula frente a su enseñanza, sino también en la forma cómo los estudiantes aprenden, en lo que eligen aprender y a qué ritmo, entre otros factores.

Por consiguiente, la investigación sobre la intersección entre metacognición y personalidad nunca ha sido más apremiante. El presente estudio no solo muestra que las características de la personalidad están relacionadas con las habilidades metacognitivas, sino que también evidencia que las más relevantes para ciertas habilidades metacognitivas, a saber, el conocimiento declarativo, el conocimiento condicional, la planificación y el monitoreo, parecen ser la responsabilidad y, en menor medida, la extraversión.

Estos hallazgos exploratorios sugieren tentativamente que las habilidades metacognitivas de los individuos pueden estar influenciadas, aunque sea levemente, por los rasgos de personalidad. Esto tiene una importancia fundamental para los investigadores en ambos ámbitos, así como para el aprendizaje de los científicos y los docentes.

Los hallazgos del presente estudio allanan el camino para exploraciones adicionales sobre cómo interactúan la personalidad (una construcción "cálida") y la metacognición (una construcción "fría"). También pueden ayudar a explicar las discrepancias e inconsistencias en hallazgos previos sobre los efectos del entrenamiento de las estrategias cognitivas en el monitoreo metacognitivo y los resultados del aprendizaje.

¿Qué pasa si las características de personalidad de alguna manera determinan cómo los maestros planifican, desarrollan o reflexionan sobre su enseñanza, o acerca de cómo los 
estudiantes reciben estas intervenciones educativas?, ¿qué pasaría si estas intervenciones previamente generales, pudieran adaptarse a fin de ser más interesantes para las personas según los perfiles de personalidad?, ¿se podrían utilizar estos perfiles de personalidad en conjunto con los perfiles de estilos metacognitivos para satisfacer mejor las necesidades de aprendizaje de los individuos (es decir, estar más en sintonía con las diferencias individuales en el aprendizaje frente al gran énfasis en las normas grupales y las evaluaciones estandarizadas)?

Por lo tanto, aunque de manera tentativa y exploratoria, los hallazgos que se presentan tienen cierta relación con la investigación en ciernes sobre la intersección entre la personalidad y la (meta)cognición.

\section{Avenidas para la investigación futura}

Los resultados del presente estudio representan una exploración en el ámbito de las posibilidades al investigar dos constructos que, cuando se combinan, evidencian relaciones que aún no se comprenden muy bien, debido a esto los hallazgos encontrados son muy interesantes. Aunque el tamaño de la muestra es relativamente grande en comparación, la investigación futura debería replicar este estudio con tamaños de muestra más grandes para garantizar que el patrón correlacional y predictivo encontrado sea estable y consistente en múltiples muestras.

Los resultados encontrados, evidencian la necesidad de realizar estudios multiculturales para investigar hasta qué punto los resultados del presente estudio se generalizan en otras culturas. Esto es especialmente relevante, porque la cultura probablemente ejerce una gran influencia en el desarrollo de la personalidad. Algunas culturas, por ejemplo, pueden valorar más la extraversión sobre otros factores, mientras que otras valoran la apertura sobre la amabilidad. Si bien en el presente estudio se investigó la relación entre las habilidades metacognitivas (el conocimiento metacognitivo: declarativo, de procedimiento y condicional; y la regulación metacognitiva: planificación, monitoreo y evaluación) y las características de la personalidad (extraversión, amabilidad, responsabilidad, neuroticismo y apertura) utilizando encuestas de autoinforme, no empleó resultados de aprendizaje como medidas objetivas de rendimiento. La investigación futura debería, por lo tanto, examinar cómo se cruzan la personalidad, la metacognición y los resultados de aprendizaje relevantes para los educadores.

\section{Reflexiones metodológicas y limitaciones}

La presente investigación que involucró participantes humanos no está exenta de limitaciones. Primero, este estudio representa un diseño de investigación no experimental con muestreo de conveniencia. Esto limita las inferencias y conclusiones que pueden extraerse de los datos y también limita la generalización de los hallazgos encontrados a otras muestras de la misma población. 
En segundo lugar, la presente investigación empleó encuestas de autoinforme para recopilar datos, lo que representa una limitación por el sesgo de deseabilidad social propio de este tipo de instrumentos psicométricos. En el presente estudio se consideró que los participantes se calificaron a sí mismos altamente entre las variables incluidas en el estudio y, por lo tanto, potencialmente pudieron haber creado efectos de techo. Esto también limita las inferencias y conclusiones que uno puede sacar de los resultados que se presentan.

Finalmente, es importante reconocer que los tamaños del efecto para los modelos de regresión fueron todos pequeños $y$, en consecuencia, el lector debe interpretar la importancia práctica de los hallazgos presentados considerando esto. Claramente, hay otros factores, como la motivación, el estilo atribucional, los incentivos, etc., que no se tuvieron en cuenta en los modelos que se analizaron y que contribuyen a la predicción de estas variables metacognitivas, aunque, dada la naturaleza exploratoria del presente estudio, esto no es tan problemático.

A pesar de las diversas limitaciones de la presente investigación, se considera esencial subrayar dos puntos fuertes. Primero, la investigación se realizó en un entorno ecológicamente válido (es decir, en aulas reales en las que los estudiantes aprendieron) en lugar del entorno artificial de un laboratorio y, por consiguiente, las conclusiones que se presentan son más válidas contextualmente. Segundo, se realizó una relación entre variables en si misma innovadora desde el punto de vista teórico e investigativo, al ser un estudio en el que se exploraron las relaciones entre las variables de personalidad y metacognitivas, tomando en cuenta que existe poca investigación hasta la fecha sobre estas relaciones. En tal sentido, se considera que los hallazgos presentados contribuyen sustancialmente a mejorar las explicaciones a cerca de la compleja relación entre estas dos variables: la personalidad y la (meta)cognición.

\section{Conclusión}

En esta investigación se exploró la relación entre las características de la personalidad y las habilidades metacognitivas medidas por el AEP y el MAIT, respectivamente. Entre los resultados encontrados, se descubrió que la personalidad y la metacognición están relacionadas a lo largo de líneas teóricamente defendibles. Se encontraron correlaciones de pequeñas a moderadas entre la mayoría de las variables de personalidad y metacognitivas. También se encontró apoyo parcial para la hipótesis inicial del estudio, en la medida en la que solo la responsabilidad, como factor de personalidad, predijo significativamente el conocimiento declarativo, el conocimiento condicional, la planificación y el monitoreo. Aunque la presente investigación representa un estudio exploratorio, descriptivo e inferencial basado en medidas de autoinforme, el estudio presentado marca el primero, del que se tenga conocimiento, que investiga estos dos temas sistemáticamente en habla hispana. Si bien la intersección entre la personalidad y la metacognición es ciertamente mucho más compleja de lo que sugieren los resultados 
presentados, se espera que estos hallazgos inicial es estimulen lo que se espera, que sea un programa productivo de estudio sobre la relación dinámica entre la personalidad, la metacognición y el aprendizaje.

\section{Referencias}

Adadan, E., \& Oner, D. (2018). Examining preservice teachers' reflective thinking skills in the context of web-based portfolios: The role of metacognitive awareness. Australian Journal of Teacher Education, 43(11), 26-50. https://search.informit.com.au/documentSummary; dn=107182555493753;,res=IEL APA

Ardi, A., Fadilah, M., \& Ichsani, B. (2018). Metacognitive ability relationship with rest result of senior high school of biology teacher competence in Sijunjung District. IOP Conf. Series: Materials Science and Engineering, (335), 1-7. https://dx.doi.org/10.1088/1757-899X/335/1/012086

Balcikanli, C. (2011). Inventario de Conciencia Metacognitiva para Docentes (MAIT). Electronic Journal of Research in Educational Psychology, 9(3), 1309-1332. http://dx.doi.org/10.25115/ejrep.v9i25.1620

Batteson, T., Tormey, R., \& Ritchiec, T. (2014). Approaches to learning, metacognition and personality; an exploratory and confirmatory factor analysis. Procedia. Social and Behavioral Sciences, (116),

2561-2567.

https://dx.doi.org/10.1016/j.sbspro.2014.01.611

Bidjerano, T., \& YunDai, D. (2007). The relationship between the big-five model of personality and self-regulated learning strategies. Learning and Individual Differences (17), 69-81. https://dx.doi.org/10.1016/j.lindif.2007.02.001

Blair, C., Calkins, S., \& Kopp, L. (2010). Self-regulation as the interface of emotional and cognitive development: Implications for education and academic achievement. In R. H. Hoyle (Ed.), Handbook of personality and self-regulation (pp. 64-90). Malden, MA: Blackwell Publishing Ltd.

Bol, L., Hacker, D. J., O'Shea, P., \& Allen, D. (2005). The influence of overt practice, achievement level, and explanatory style on calibration accuracy, and performance. The Journal of Experimental Education, (73), 269-290. https://doi.org/10.3200/JEXE.73.4.269-290

Burson, K. A., Larrick, R. P., \& Klayman, J. (2006). Skilled or unskilled, but still unaware of it: How perceptions of difficulty drive miscalibration in relative comparisons. Journal of Personality and Social Psychology, 90(1), 60-77. https://dx.doi.org/10.1037/0022-3514.90.1.60 
Cohen, J. (1988). Statistical power analysis for the behavioral sciences ( $2^{\text {nd }}$ ed.). Lawrence Earlbaum Associates. https://doi.org/10.1016/C2013-0-10517-X

Congreso de la República de Colombia. (2012, 17 de octubre). Ley 1581. http://wsp.presidencia.gov.co/Normativa/Leyes/Documents/LEY\%201581\%20DEL \%2017\%20DE\%200CTUBRE\%20DE\%202012.pdf

Costa, P., Terracciano, A., \& McCrae, R. (2001). Gender differences in personality traits across cultures: Robust and surprising findings. Journal of Personality and Social Psychology, (81), 322-331. https://dx.doi.org/10.1037/0022-3514.81.2.322

Dörrenbächer, L., \& Perels, F (2016). Self-regulated learning profiles in college students: Their relationship to achievement, personality, and the effectiveness of an intervention to foster self-regulated learning. Learning and Individual Differences, 51, 229-241. https://dx.doi.org/10.1016/j.lindif.2016.09.015

Fathima, M., Sasikumar, N., \& Roja, M. (2014). Enhancing teaching competency of graduate teacher trainees through metacognitive intervention strategies. American Journal of Applied Psychology, 2(1), 27-32. https://dx.doi.org/10.12691/ajap-2-1-5

Goldberg, L. (1992). The development of markers for the Big-Five factor structure. Psychological Assessment, (4), 26-42. https://dx.doi.org/10.1037/10403590.4.1.26

Goldberg, L. (1993). The structure of phenotypic personality traits. American Psychologist, (48), 26-34. https://dx.doi.org/10.1037/0003-066X.48.1.26

Goldberg, L., Johnson, J., Eber, H., Hogan, R., Ashton, M., Cloninger, R., \& Gough, H. (2006). The international personality item pool and the future of public-domain personality measures. Journal of Research in Personality, (40), 84-96. https://doi.org/10.1016/j.jrp.2005.08.007

Ghonsoolya, B., Hassan Khajavyb, G., \& MohagheghMahjoobi, F. (2014). Self-efficacy and metacognition as predictors of Iranian teacher trainees' academic performance: A path analysis approach. Procedia. Social and Behavioral Sciences, (98), 590-598. https://dx.doi.org/10.1016/j.sbspro.2014.03.455

Gutierrez, A. P., \& Schraw, G. (2015). Effects of strategy training and incentives on students' performance, confidence, and calibration. Journal of Experimental Education, 83(3), 386-404. https://dx.doi.org/10.1080/00220973.2014.907230

Gutierrez de Blume, A. P. (2017). The effects of strategy training and an extrinsic incentive on fourth-and fifth-grade students' performance, confidence, and calibration accuracy. Cogent Education, (4), 1-17. https://dx.doi.org/10.1080/2331186X.2017.1314652 
Hogan, R., Hogan, J., \& Roberts, B. W. (1996). Personality measurement and employment decisions: Questions and answers. American Psychologist, (51), 469-477. https://dx.doi.org/10.1037/0003-066X.51.5.469

Jiang, Y., Ma, L., \& Gao, L. (2016). Assessing teachers' metacognition in teaching: The teacher Metacognition Inventory. Teaching and Teacher Education, (59), 403-413. https://dx.doi.org/10.1016/j.tate.2016.07.014

Joseph, N. (2009). Metacognition needed: Teaching middle and high school students to develop strategic learning skills. Preventing School Failure: Alternative Education for Children and Youth, 59-103. https://dx.doi.org/10.1080/10459880903217770

Kallio, H., Virta, K., Kallio, M., Virta, A., Hjardemaal, F., \& Sandven, J. (2017). The utility of the metacognitive awareness inventory for teachers among in-service teachers. Journal of Education and Learning, 6(4), 78-91. https://dx.doi.org/10.5539/jel.v6n4p78

Kim, L., Jörg, V., \& Klassen, R. (2019). A Meta-Analysis of the Effects of Teacher Personality on Teacher Effectiveness and Burnout. Educational Psychology Review, (31), 163-195. https://doi.org/10.1007/s10648-018-9458-2

Kramarski, B., \& Michalsky, T. (2009). Investigating preservice teachers' professional growth in self-regulated learning environments. Journal of educational psychology, 101(1), 161-175. http://dx.doi.org/10.1037/a0013101

Komarraju, M., Karau, S., Schmeck, R., \& Avdic, A. (2011). The Big Five personality traits, learning styles, and academic achievement. Personality and Individual Differences 51: 472-477. https://dx.doi.org/10.1016/j.paid.2011.04.019

Ledesma, R., Sánchez, R., \& Díaz-Lázaro, C. (2011). Adjective checklist to assess the Big Five personality factors in the Argentine population. Journal of Personality Assessment, 93(1), 46-55. https://dx.doi.org/10.1080/00223891.2010.513708

McCrae, R., \& Costa, P. (1987). Validation of the five-factor model of personality across instruments and observers. Journal of Personality and Social Psychology, 52(1), 8190. https://dx.doi.org/10.1037//0022-3514.52.1.81

Mai, M. (2015). Science teachers' self-perception about metacognition. Journal of Educational and Social Research, 571), https://dx.doi.org/10.5901/jesr.2015.v5n1s1p77

Maltby, J., Day, L., \& Macaskill, A. (2007). Personality, individual differences and intelligence ( $2^{\text {nd }}$ ed.). Estados Unidos: Prentice Hall.

Ministerio de Salud República de Colombia. (1993). Resolución 008430 del 4 de octubre. https://www.minsalud.gov.co/sites/rid/Lists/BibliotecaDigital/RIDE/DE/DIJ/RESOLU CION-8430-DE-1993.PDF 
Nietfeld, J., \& Schraw, G. (2002). The Effect of Knowledge and Strategy Training on Monitoring Accuracy, The Journal of Educational Research, 95 (3), 131-142. https://doi.org/10.1080/00220670209596583

Ozturk, N. (2020). An Analysis of Teachers' Metacognition and Personality. Psychology and Education, 57(1), 40-44. http://www.psychologyandeducation.net/Article/ananalysis-of-teachers-metacognition-and-personality.pdf

Öz ${ }^{\mathrm{a}}$, H. (2016). The importance of personality traits in students' perceptions of metacognitive awareness. Procedia. Social and Behavioral Sciences, (232), 655667. https://dx.doi.org/10.1016/j.sbspro.2016.10.090

Palantis, N., Mohamed, J., Ibrahim, A., Ismail, S., Anuar, N., Ma'rof, A., \& Buang, N. (2017). Patterns of metacognitive awareness among primary school teachers. Jurnal Varidika. Kajian Penelitian Pendidikan, 29(2), 141-146. http://journals.ums.ac.id/index.php/varidika/article/view/5629/3675

Pallier, G., Wilkinson, R., Danthiir, V., Kleitman, S., Knezevic, G., Stankov, L., et al. (2002). The role of individual differences in the accuracy of confidence judgments. The Journal of General Psychology, 129(3), 257-299. https://dx.doi.org/10.1080/00221300209602099

Prytula, M. P. (2012). Teacher metacognition within the professional learning community. International Education Studies, 5(4), 112-121. https://dx.doi.org/10.5539/ies.v5n4p112

Rahman, F. U. (2011). Assessment of science teachers' metacognitive awareness and its impact on the performance of students (Doctoral dissertation, ALLAMA IQBAL Open University, Islamabad). http://prr.hec.gov.pk/jspui/handle/123456789/1076

Sanmartí, N. (2008). 10 ideas clave. Evaluar para aprender. Editorial Graó.

Sánchez, R., \& Ledesma, R. ( 2007). Los cinco grandes factores : cómo entender la personalidad y cómo evaluarla . En A. Monjeau (Ed.), Conocimiento para la transformación. (Serie Investigación y Desarrollo , pp. 131-160). Ediciones Universidad Atlántida Argentina.

Sánchez, R., \& Ledesma, R. (2013). Listado de adjetivos para evaluar personalidad: Propiedades y normas para una población argentina. Revista Argentina de Clínica Psicológica, 22(2), 147-160. https://www.revistaclinicapsicologica.com/busqueda_de_trabajos.php

Stankov, L., Morony, S., \& Ping Lee, Y. (2014). Confidence: the best non-cognitive predictor of academic achievement? Educational Psychology. An International Journal of Experimental Educational Psychology, 34(1), 9-28. https://dx.doi.org/10.1080/01443410.2013.814194 
Sawyer, K. (2014). The Cambridge handbook of the learning sciences. Cambridge University Press. https://doi.org/10.1017/CBO9780511816833

Schraw, G., \& Dennison, R. (1994). Assessing metacognitive awareness. Contemporary Educational Psychology, (19), 460-475. https://dx.doi.org/10.1006/ceps.1994.1033

Stephenson, V. (2008). From the beginning: introducing metacognition and thinking skills in initial teacher education. Gifted Education International, (24), 283-284. https://dx.doi.org/10.1177\%2F026142940802400319

Thiede, K., Oswalt, J., Bredefur, M., Osguthorpe, C., \& Osguthorpe, M. (2019). Teachers' judgments of student learning of mathematics. In, The Cambridge handbook cognition and education (pp. 678-694). Cambridge University Press. https://doi.org/10.1017/9781108235631.027

Wilson, N., \& Bai, H. (2010). The relationships and impact of teachers' metacognitive knowledge and pedagogical understandings of metacognition. Metacognition Learning, 5, 269-288. https://doi.org/10.1007/s11409-010-9062-4

Zimmerman, B. J., \& Moylan, A. R. (2009). Self-regulation: Where metacognition and motivation intersect. In D. J. Hacker, J. Dunlosky\& A. C. Graesser (Eds.), Handbook of Metacognition in Education (pp. 299- 315). New York: Routledge.

Zohar, A. (2006). The nature and development of teachers' metastrategic knowledge in the context of teaching higher order thinking. The Journal of Learning Sciences, 15(3), 331-377. https://dx.doi.org/10.1207/s15327809jls1503_2

Zohar, A., \& Dori, Y. (2012). Metacognition in science education. Springer. https://doi.org/10.1007/978-94-007-2132-6 